\title{
A DUCK WITH FOUR LEGS: INVESTIGATING THE STRUCTURE OF CONCEPTUAL KNOWLEDGE USING PICTURE DRAWING IN SEMANTIC DEMENTIA
}

\author{
Sasha Bozeat \\ MRC Cognition and Brain Sciences Unit, Cambridge, UK \\ Matthew A. Lambon Ralph \\ Manchester University, UK \\ Kim S. Graham and Karalyn Patterson \\ MRC Cognition and Brain Sciences Unit, Cambridge, UK \\ Helen Wilkin and Josephine Rowland \\ University of Cambridge, UK \\ Timothy T. Rogers \\ $M R C$ Cognition and Brain Sciences Unit, Cambridge, UK \\ John R. Hodges \\ MRC Cognition and Brain Sciences Unit and Addenbrooke's Hospital, Cambridge, UK
}

\begin{abstract}
In Study 1, six patients with semantic dementia were asked to produce drawings of concrete concepts from dictation of their names. The drawings were characterised by a loss of distinctive features. In the artefact domain, this feature loss resulted in representations that were increasingly box-like. In the living domain, as well as distinctive features being lost, there was a tendency for patients to include incorrect features that resulted in more familiar and "prototypical" representations.

A second study included two further conditions in the drawing assessment: immediate and delayed copying of line drawings of concrete concepts. Analysis of the drawings produced by three patients with semantic dementia confirmed that overall performance was significantly influenced by the task condition (immediate>delayed) and severity of disease. The rate of intruding features, but not of omitted ones, was influenced by the domain of the item, with a greater proportion of intrusions in the living than in the nonliving domain. There was also a significant effect of feature distinctiveness on the proportions of these error types: Intruded features were most likely to come from the pool of properties that are shared across domain.
\end{abstract}

\section{INTRODUCTION}

Semantic memory encompasses our knowledge about objects, facts, and concepts, as well as words and their meaning. The primary aim of this study was to extend previous investigations of the structure and internal representation of visual conceptual knowledge by analysing the rich database

Requests for reprints should be addressed to Sasha Bozeat, MRC Cognition and Brain Sciences Unit, Elsworth House, Box 58, Addenbrooke's Hospital, Cambridge CB2 2EF, UK (Tel: +(0) 1223 355294; Fax: +(0) 1223 359062;

Email: sasha.bozeat@mrc-cbu.cam.ac.uk). 
afforded by analysis of pictures drawn by patients with semantic dementia. The organisation of the semantic system can be revealed by studying the breakdown of conceptual knowledge observed in diseases such as herpes simplex virus encephalitis (HSVE), and Alzheimer's disease (Garrard, Patterson, Watson, \& Hodges, 1998; Lambon Ralph, Patterson, \& Hodges, 1997; Sartori, Job, Miozzo, Zago, \& Marchioni, 1993; Warrington \& Shallice, 1984). The syndrome of semantic dementia, associated with circumscribed temporal lobe atrophy, is characterised by a very selective impairment of conceptual knowledge, making it a particularly informative testing ground for investigating the structure of the semantic system (Hodges, Garrard, \& Patterson, 1998; Warrington, 1975).

\section{What can semantic dementia reveal about the structure of the semantic system?}

In previous studies, a variety of different tasks have been used to explore the nature of the semantic deficit in semantic dementia and, therefore, the architecture of the semantic memory system. We have reported impairments on tasks ranging from sound recognition to the production of names in response to definitions, with strong item consistency in performance across different modalities tested (Bozeat, Lambon Ralph, Garrard, Patterson, \& Hodges, 2000). This evidence suggests that semantic dementia is best characterised as a progressive deterioration of the conceptual knowledge that supports semantic performance, independent of modality (Bozeat et al., 2000; Lambon Ralph \& Howard, 2000) and accords with proposals that conceptual knowledge is underpinned by a unitary, central system (Caramazza, Hillis, Rapp, \& Romani, 1990). Warrington (1975) argued that semantic memory is hierarchically organised, with superordinate distinctions (e.g., between living things and artefacts), basic level categories (e.g., animals and tools) and individual exemplars represented at different levels. Some observations are in apparent accord with this view, for example, a longitudinal study of object naming in semantic dementia in which the pattern of errors suggested earliest degradation to parts of the hierarchy corre- sponding to specific concepts (e.g., time 1: duck $\rightarrow$ "duck", time 2: duck $\rightarrow$ "swan", time 3: duck $\rightarrow$ "bird", time 4: duck $\rightarrow$ "animal"; the four time periods spanned 18 months; Hodges, Graham, \& Patterson, 1995). Further relevant evidence comes from performance on tasks like word and picture sorting, in which the ability to assign concepts to their superordinate categories invariably outlasts the ability to draw distinctions based on more specific criteria (Hodges, Patterson, \& Tyler, 1994).

Although this view does have intuitive appeal, there are several observations about the nature of categories and concepts that suggest the need for a more complex view of the structure of conceptual knowledge. First, some concepts are much more typical of their categories than others (Rosch, 1973). Second, in some cases, category membership may be unclear or items may seem to belong to more than one category. Last, some categories appear to be much more coherent than others, for example, "vehicles" versus "household items."

Another way of thinking about this issue is that categories are products of individual representations that share a partially common set of features. Some studies have attempted to explore this approach to the basis of conceptual knowledge by asking normal participants to produce semantic features for a selection of concrete exemplars. Analyses of these databases suggest that higherorder structures emerge readily from a distributed collage of these fine-grained attributes. Broad conceptual domains and categories are formed from the similarity in feature structure amongst groups of concepts, without explicit coding of category membership (Garrard, Lambon Ralph, Hodges, \& Patterson, 2001; McRae \& Cree, 2002). Variations in typicality are also captured by this approach. Garrard et al. demonstrated that rated prototypicality was reliably predicted by feature similarity between a specific exemplar and the average, or centroid, of all the concepts in that category. The same structure is extracted when semantic features and other fine-grained information are incorporated into connectionist networks, which also allow a direct comparison with patient performance following simulated damage. Using this type of network, Rogers, Lambon Ralph, Patterson,

\section{COGNITIVE NEUROPSYCHOLOGY, 2003, 20 (1)}


Bozeat, McClelland, and Hodges (2002a) were able to reproduce, for example, the hierarchical effect observed in semantic dementia (Hodges et al., 1995; Warrington, 1975), without explicit coding of a hierarchical structure.

Feature listing studies have also revealed interesting differences in the distribution of shared and distinctive attributes across the different categories (see Table 6 in McRae \& Cree, 2002). Features can vary along a shared-distinctive continuum such that some attributes are relatively specific to a single exemplar (e.g., the iridescent green feathers on a male mallard), while other attributes of the same exemplar can be true of most concepts within the category or domain (e.g., the wings on a duck). Analyses of feature databases consistently show that shared features are generally more common in the living than the nonliving domains (Devlin, Gonnerman, Andersen, \& Seidenberg, 1998; Garrard et al., 2001; McRae \& Cree, 2002; Rogers et al., 2002a). As will become apparent later, this uneven distribution of shared features, as well as other structural characteristics of conceptual representation noted above, are reflected directly in drawings by patients with semantic dementia.

\section{Using drawing as an assessment of semantics}

As discussed earlier, our previous studies have used a variety of different assessments to investigate the nature of the semantic deficit in semantic dementia. The purpose of the studies reported here was to explore the use of drawing assessments as an additional line of evidence in our quest for understanding the structure and internal architecture of conceptual knowledge in the semantic system.

Drawing is widely used as a clinical assessment for investigating such disorders as constructional apraxia or neglect, and is included in a number of standard neuropsychological tests, for example, the Mini-Mental State Examination (Folstein, Folstein, \& McHugh, 1975). Drawing may also provide a rich and sensitive way of assessing peoples' knowledge about concepts, a kind of nonverbal counterpart to the feature listing technique. Drawing avoids the problem of anomia in feature listing or definition tasks, is easy to administer, requires no complex instructions, and provides a rich data set. There are, however, two potential drawbacks. First, people have very different premorbid drawing skills and this must be taken into account when evaluating impaired performance. The second problem is how to score the drawings. The standard measure, in which normal participants are asked to rate how good they think a drawing is, is an efficient method of scoring large numbers of drawings, but may be influenced by the degree of the producer's drawing skill. It also fails to allow more detailed analysis of the content of each depiction. In Study 1, we adopted and improved an alternative method used in a previous single-case study of a patient with semantic dementia (Lambon Ralph, Howard, Nightingale, \& Ellis, 1998). Normal participants were asked to produce drawings of the items, from which a list of integral features can be formulated for each item. Patient drawings can then be scored in terms of the number of target features present and compared to the range of performance shown by control subjects. The method provides, therefore, a relatively objective score that focuses on the content of each drawing and minimises the influence of drawing skill.

\section{STUDY 1}

\section{Method}

Subjects

Eight normal participants were selected from the Medical Research Council Cognition and Brain Sciences Unit's participant panel. There were five male and three female subjects, between the ages of 55 and 73 (mean $=61.75 ; S D=6.54)$.

\section{Control data collection}

The control subjects were asked to draw from name the 64 items selected from the corpus of Snodgrass and Vanderwart (1980) corresponding to the concepts included in the semantic memory test battery (see below). The order of presentation alternated between living and man-made concepts. The subjects were given $1 \mathrm{~min}$ to draw each item and were told that their drawings would not be assessed 
for artistic ability but only for representing the nature of the object.

\section{Scoring scheme}

Two independent raters (HW and JR) examined these control drawings and assembled a list of all features present for each item. For example, for a duck the list included the following properties: body, head, 2 legs, webbed feet, beak, and wings. For each item, each of the eight control depictions was compared to this list and features present in six or more of the drawings were included in the final scoring scheme. These target features reflect the attributes that the majority of normal subjects include in their drawings, irrespective of drawing skill or other characteristics that may vary across individuals. Note that the maximum possible score for each item varied depending on the number of features produced in the control drawings. Three items (plug, barrel, and alligator) had to be excluded from the analysis as the names were either misheard or misunderstood by a number of the control subjects.

\section{Patients}

Six patients were identified through the Memory and Cognitive Disorders Clinic at Addenbrooke's Hospital, Cambridge, UK, where they were seen by a senior neurologist (JRH), a senior psychiatrist, and a clinical neuropsychologist. There were two male and four female subjects, between the ages of 57 and 61 years (see Table 1 ). In addition to a clinical assessment, all patients were given a number of standard psychiatric rating scales to exclude major functional psychiatric disorders such as depression and schizophrenia. They all underwent MRI scan- ning together with the usual battery of screening blood tests to exclude treatable causes of dementia.

All patients presented with a progressive loss of vocabulary affecting expressive and receptive language in the context of fluent speech production. They all fulfilled the criteria for semantic dementia previously reported (Hodges et al., 1995; Hodges, Patterson, Oxbury, \& Funnell, 1992). Structural brain imaging by MRI showed focal atrophy involving the polar and inferolateral regions of one or both of the temporal lobes (see Table 1 for further details).

\section{General neuropsychology}

The following battery of neuropsychological tests was administered: the Mini-Mental State Examination as a general measure of cognitive impairment (Folstein et al., 1975); verbal fluency for the letters F, A, S to test executive function; the Raven's advanced progressive matrices or coloured matrices to assess nonverbal problem solving (Raven, 1962, 1965); copy and immediate recall of the Rey Complex Figure (Ray, 1941) to test visuospatial skills and episodic memory. Various subtests from the Visual Object and Space Perception battery were also used to assess visuospatial function in more detail (Warrington \& James, 1991).

\section{Semantic assessments}

The patients were given a selection of tasks from a semantic battery, which is a collection of tests that use the same set of stimulus items to assess semantic knowledge systematically across different input and output modalities. It contains 64 items representing three categories of living things (animals, birds, and fruit) and three categories of artefacts (house-

Table 1. Demographic and imaging data

\begin{tabular}{|c|c|c|c|c|c|c|c|}
\hline & $D M$ & $M B$ & $K H$ & $G C$ & $J H$ & $F M$ & $\begin{array}{l}\text { Control } \\
\text { mean (SD) }\end{array}$ \\
\hline Age & 61 & 58 & 57 & 59 & 58 & 59 & $61.7(6.5)$ \\
\hline Sex & M & $\mathrm{F}$ & M & $\mathrm{F}$ & $\mathrm{F}$ & $\mathrm{F}$ & $5: 3^{\mathrm{a}}$ \\
\hline MRI & Mild left & Moderate & Moderate & Moderate & Moderate & Moderate & \\
\hline & temporal atrophy & $\begin{array}{l}\text { bilateral fronto- } \\
\text { temporal atrophy } \\
L>R\end{array}$ & $\begin{array}{l}\text { bilateral temporal } \\
\text { atrophy } \\
L>R\end{array}$ & $\begin{array}{l}\text { bilateral temporal } \\
\text { atrophy } \\
L>R\end{array}$ & $\begin{array}{l}\text { bilateral temporal } \\
\text { atrophy } \\
L>R\end{array}$ & $\begin{array}{l}\text { bilateral temporal } \\
\text { atrophy } \\
L>R\end{array}$ & \\
\hline
\end{tabular}

${ }^{\mathrm{a}}$ Ratio male to female. 
hold items, tools, and vehicles). These are the same 64 exemplars that were included in the drawing assessment. The following subtests from the semantic battery were administered: category fluency, in which the subject is asked to produce as many exemplars as possible in $1 \mathrm{~min}$ for each of the 6 categories; naming of the 64 line drawings; spoken word-to-picture matching using picture arrays containing the target plus 9 within-category foils.

As an additional semantic assessment, we administered the Pyramids and Palm Trees test (Howard \& Patterson, 1992), in which subjects are asked to choose one of two items that is most closely associated with the target (e.g., for the target pyramid, the choice is between palm tree and pine tree). The stimuli are presented as either pictures or written words.

\section{Drawing assessment}

The patients were asked to produce drawings of the 64 items from their names and were given unlimited time to draw each of them. Although there is little evidence for perseverative errors in patients with semantic dementia, we tried to minimise this possibility by alternating the presentation of living and nonliving concepts and asking the patients to use a fresh sheet of paper for each drawing. The drawings produced by the patients and the control subjects were scored by two independent raters according to the feature-based scoring scheme described above.

\section{Results}

\section{General neuropsychology}

The six patients covered a broad spectrum of general impairment as indicated by their performance on the Mini-Mental State Examination (see Table 2). All patients, except for DM, exhibited some impairment on the letter fluency test. This result, however, is most likely to reflect the patients' anomia rather than any significant deficit in executive functioning. There was general preservation of nonverbal problem-solving skills, as measured by Raven's matrices, except in the case of MB, who had an unexpectedly poor performance on this test. All patients had preserved visuospatial skills as indicated by their copying of the Rey Figure and performance on the various subtests of the Visual Object and Space Perception battery, though FM performed poorly on two subtests of the VOSP. Of particular interest is the good performance on object decision: even the most impaired patients achieved scores within the normal range on the VOSP version of this task. Recall of the Rey Figure was good in most cases revealing general preservation of nonverbal episodic memory, except in the two most impaired patients, FM and JH.

Table 2. General neuropsychology

\begin{tabular}{|c|c|c|c|c|c|c|c|}
\hline Test (maximum score) & $D M$ & $M B$ & $K H$ & $G C$ & $J H$ & $F M$ & Control mean (SD) \\
\hline$M M S E(30)$ & 29 & 22 & 23 & 25 & 24 & 8 & $28.8(0.5)$ \\
\hline Letter fluency (total: FAS) & 49 & 16 & 18 & 16 & 12 & 1 & $44.2(11.2)$ \\
\hline Raven's matrices (percentiles) & $50^{\mathrm{b}}$ & $10^{\mathrm{a}}$ & $>95^{\mathrm{a}}$ & $50^{\mathrm{b}}$ & $75-90^{\mathrm{a}}$ & $50^{\mathrm{a}}$ & \\
\hline \multicolumn{8}{|l|}{ Rey Figure } \\
\hline Copy (36) & 34 & 33 & 33 & 35 & 36 & 30 & $34(2.9)$ \\
\hline Immediate recall (36) & 26 & 13 & 16.5 & 10 & 9 & 6 & $18.3(5.2)$ \\
\hline \multicolumn{8}{|l|}{ VOSP } \\
\hline Incomplete letters (20) & 19 & 19 & 20 & 20 & 19 & NT & $19.2(0.8)$ \\
\hline Dot counting (10) & 10 & 9 & 10 & 10 & 10 & 10 & $9.9(0.3)$ \\
\hline Object decision (20) & 17 & 14 & 19 & 14 & 15 & 15 & $16.5(3.5)$ \\
\hline Position discrimination (20) & 20 & 20 & 19 & 20 & 20 & 15 & $19.8(0.6)$ \\
\hline Cube analysis (10) & 10 & 7 & 10 & 8 & 10 & 4 & $9.792 .5)$ \\
\hline Number location (10) & 10 & 8 & 10 & 10 & 10 & 5 & $8.9(2.8)$ \\
\hline
\end{tabular}

The patients are ordered according to their performance on the naming test (see Table 3). VOSP: Visual Object and Space Perception battery; NT: not tested.

${ }^{\mathrm{a}}$ Raven's coloured matrices; ${ }^{\mathrm{b}}$ Raven's advanced progressive matrices. 
BOZEAT ET AL.

Table 3. Assessment of semantic memory

\begin{tabular}{|c|c|c|c|c|c|c|c|}
\hline Test (maximum score) & $D M$ & $M B$ & $K H$ & $G C$ & $J H$ & $F M$ & Control mean (SD) \\
\hline \multicolumn{8}{|l|}{ Category fluency } \\
\hline Living & 53 & 23 & 11 & 15 & 7 & 9 & $60.3(12.6)$ \\
\hline Man-made & 54 & 12 & 25 & 3 & 7 & 0 & $54.8(10.3)$ \\
\hline Naming (64) & 61 & 55 & 49 & 27 & 10 & 0 & $62.3(1.6)$ \\
\hline Word-picture matching (64) & 63 & 59 & 58 & 42 & 27 & 16 & $63.7(0.5)$ \\
\hline \multicolumn{8}{|l|}{ Pyramids and Palm Trees } \\
\hline Words (52) & 43 & 43 & 50 & 35 & 31 & NT & $51.1(1.1)$ \\
\hline Pictures (52) & 46 & 42 & 45 & 35 & 40 & 34 & $51.2(1.4)$ \\
\hline
\end{tabular}

The patients are ordered according to their performance on the naming test. NT: not tested.

\section{Assessment of semantics}

As shown in Table 3, the patients included in this study also covered a broad range of semantic decline from the mildly impaired patient DM through to FM, who was severely impaired. It should be noted that although DM showed mild impairment on the Pyramid and Palm Trees tests only, follow-up testing has revealed a steady decline leading to impairment on all other semantic assessments consistent with a diagnosis of semantic dementia. All patients, except for $\mathrm{DM}^{1}$, showed reduced category fluency, a mild to severe anomia, and impaired comprehension as measured by word-to-picture matching (NB, the patients are ordered by their naming and comprehension scores in Tables 2 and 3). All patients were impaired on both conditions of the Pyramid and Palm Trees test.

\section{Analysis of drawings}

Reliability between the two raters was confirmed using Cohen's Kappa analysis for the drawings of both the patients $(\mathrm{k}=.42, p<.05)$ and the controls $(\mathrm{k}=.48, p<.05)$. The raters' two scores for each item were averaged and converted to reflect the proportion of target features present in each depiction.

A Mann-Whitney $U$ test confirmed that the patients produced a significantly lower proportion of target features than the control subjects: $\mathrm{U}=1.5$, $p<.01$ (patient mean $(S D)=0.53(0.31)$; control mean $(S D)=0.95(0.04))$.

\section{Comparison of drawing with performance on naming and word-to-picture matching}

As noted in the Introduction, previous results from studies of semantic dementia suggest that the patients' performance on the drawing assessment should reflect impairment to a single central semantic system. If this is the case, performance on the drawing assessment should be highly correlated with performance on the other semantic tests.

Spearman's correlations revealed significant associations between the patients' performance on the naming test, the word-to-picture matching test, and the drawing assessment $(r$ between .96 and .99 , all $p<.001$, one-tailed). Inspection of the patients' performance on other general neuropsychological tasks reveals that, whilst performance on general semantic tasks declines, performance on other visual and problem-solving tasks such as copying the Rey figure (Rey, 1941) and the Raven's coloured matrices (Raven, 1962) is relatively well-preserved until very late in the course of the disease. These results not only confirm that the patients suffer from a selective and progressive semantic impairment (Hodges et al., 1992; Warrington, 1975), but are also compatible with the hypothesis that their poor drawing is due entirely to impoverished conceptual representations.

Multiple regression analyses were used to investigate the influence of familiarity of the item and the domain (living or nonliving) on the patients' drawings, for the patients' individual scores, and

\footnotetext{
${ }^{1}$ Although DM was mildly affected at this stage, his good performance on these spoken output tasks may be due, at least in part, to his daily vocabulary practice routines (see Graham, Patterson, Pratt, \& Hodges, 1999).
} 
also on the average patient score for each item. In the group analysis, there was a trend for better drawings for the more familiar items $(t=1.78, p=$ $.07)$, with two individual patients showing the same trend (DM: $t=1.71, p=0.09 ; \mathrm{FM}: t=1.77, p=.08$ ). The performance of one patient $(\mathrm{KH})$ was significantly influenced by the domain of the item, in favour of the man-made items $(t=5.4, p<.001)$.

\section{Qualitative observations}

Five of the patients produced some drawings that were recognisable as items other than those they had been asked to produce (these drawings were scored in the same way as the other items). Most of these errors were semantically related (e.g., "watering can" $\rightarrow$ hose; "kangaroo" $\rightarrow$ giraffe). Crossdomain errors were extremely rare and only observed in two patients (JH and GC) who both had a fairly long disease history. In addition, there were some instances where patients refused to draw an item because they had no idea what the name meant.

As noted previously by Lambon Ralph et al. (1998), the types of drawing errors varied across domain. For living concepts, there was both a loss of distinctive features and a tendency to produce a representation that was highly familiar and "prototypical" of this domain. In some cases, incorrect features were added on, for example, four legs on a duck. In the artefact domain, there was a similar loss of distinctive features but incorrect features very rarely intruded. The representations tended to become much simpler and more box-like (see Figure 1).
These qualitative observations will be investigated further in Study 2 using quantitative analysis.

\section{DISCUSSION}

This first study, using feature analysis as a method of scoring the data, revealed, not surprisingly, that patients with semantic dementia produced significantly less correct information than control subjects in their drawings of objects to the dictated names. More importantly, there were substantial and significant correlations between performance on the drawing assessment, object naming, and word-topicture matching, suggesting that all of these impairments are due to selective damage to central conceptual knowledge. Overall, there was a trend towards a significant impact of item familiarity on success in drawing, as noted previously for a range of semantic tasks in semantic dementia (Bozeat et al., 2000; Funnell, 1995; Lambon Ralph et al., 1997).

In many of the drawings of concepts from both domains, we observed a loss of distinctive features leading, in the artefact domain, to representations becoming increasingly box-like. In the living domain, additional intruding features resulted in drawings that were more "prototypical" than the target items. This pattern of domain differences is similar to the longitudinal naming errors observed previously in semantic dementia (Hodges et al., 1995), and we suggest that it may result from inherent differences in the distribution of features in the two domains (McRae \& Cree, 2002; Rogers et al., 2002a). If features shared across many exemplars are more likely to be preserved, whereas distinctive

\begin{tabular}{|l|c|c|}
\hline Spoken name & Duck & Lamp \\
\hline Drawing & \\
\hline
\end{tabular}

Figure 1. Examples of drawings by $\mathrm{JH}$ - a duck and a lamp. 
features limited to a small number of concepts are more vulnerable, then the greater number of shared features for living things would tend to yield this prototypicality effect for living things. In addition, largely shared features (e.g., four legs for animals) are more likely to intrude into representations of concepts from that domain which do not properly include that feature (e.g., duck).

Although the drawing assessment used in Study 1 has a nonverbal output, it does rely on verbal input (the spoken name of each concept). We did encounter some instances where patients refused to draw an item because they had no idea what the name meant. In the second study, we adopted two methods in an attempt to minimise the rate of these omission errors. First, we employed nonperceptual prompts when a patient refused to draw an item in response to its name (Lambon Ralph et al., 1998). The second method used two additional, purely nonverbal forms of drawing assessment-immediate and delayed copying (Franklin, Van Sommers, \& Howard, 1987; Lambon Ralph et al., 1998). Semantic memory is presumed to have little or no effect upon the quality of the depiction in immediate copying, which should rely instead upon the subjects' visual, spatial, and constructional skills. If a delay is inserted between the person seeing the picture and being asked to reproduce it, however, we assume that the drawing will rely, at least in part, on the conceptual representation as well as visual memory. If central conceptual knowledge about the object is degraded, then we expect an impact on the response observed in delayed copying. The second study employed these three drawing conditionsdrawing to name, immediate copying, and delayed copying. The scoring scheme was modified such that, in addition to quantifying correct features that were either maintained or lost, it also allowed analysis of features that were incorrectly added.

\section{STUDY 2}

\section{Method}

\section{Subjects}

Three patients with semantic dementia were identified and assessed in the same way as the patients in Study 1. There were two male subjects and one female subject, between the ages of 66 and 77 years (see Table 4). To compare the performance of the patients with that of age- and education-matched controls we selected four normal participants from the Medical Research Council Cognition and Brain Sciences Unit's participant panel.

\section{Assessments}

General neuropsychology and assessment of semantics. The same general neuropsychological tests and battery of semantic assessments described in Study 1 were included here. We also administered the Digit Span subtest of the Wechsler Memory ScaleRevised (WMS-R; 1987) and the Concrete and Abstract Word Synonyms (Warrington, McKenna, \& Orpwood, 1998), which requires the subject to choose one of two words that is most similar in meaning to a target word.

Drawing battery. The subjects were asked to draw 64 items (the same as in Study 1) under three conditions (on three different occasions).

1. Immediate copying. In this condition, the subjects were allowed to study a line drawing of the

Table 4. Demographic and imaging data

\begin{tabular}{llllc}
\hline & $D S$ & $D C$ & $I F$ & Control mean (SD) \\
\hline Age & 69 & 77 & 66 & $65.5(9.5)$ \\
Sex & M & F & M & $2: 2^{\mathrm{a}}$ \\
MRI & Mild bilateral & Moderate & Marked bilateral & \\
& fronto-temporal & temporal atrophy & temporal atrophy & \\
& L $>$ R & L $>$ R & L $>$ R & \\
\hline
\end{tabular}

${ }^{\mathrm{a}}$ Ratio male-female.

\section{COGNITIVE NEUROPSYCHOLOGY, 2003, 20 (1)}


item and were asked to reproduce it with the picture still in sight.

2. Delayed copying. This technique involved showing the subjects a picture, taking it away, and asking them to count to 15 (corresponding to a delay of about $10 \mathrm{~s}$ ) before drawing the item (Franklin et al., 1987; Lambon Ralph et al., 1998). The length of delay was established through pilot testing with one patient, DS, in which different delays ranging from $10 \mathrm{~s}$ to $1 \mathrm{~min}$ were tried out with a selection of items not included in the main corpus of 64 concepts. After just 10 s delay, it was apparent that the patient's poor conceptual knowledge began to influence his delayed copying, and so this relatively short interval was selected for all further testing.

3. Drawing to name. As in Study 1, the subject was not shown a picture of the item but was instead given the name and asked to draw it. On those occasions when patients were unable to recognise the word, they were asked to draw whatever they thought the item might look like. If they were unable to do this, they were given a nonperceptual verbal prompt. For example, in the case of the duck, they would be told that it is a bird that swims on ponds, waddles on land, and lays eggs. Critically, the prompts did not contain any perceptual/structural details (e.g., it has a beak, wings, two legs, etc.).

Again we alternated the presentation of living and man-made items in all three conditions and asked the patients to use a fresh sheet of paper for each drawing.

Scoring scheme. The four control subjects were asked to draw the 64 items under the three conditions. The drawings produced under the copying condition were then examined by two independent raters (SB and TR), who compiled a list of the individual features present in the drawings of each concept. For example, in the drawing of a duck the raters listed the following features: body, head, legs (2), wings (2), beak, eyes (2), tail (short, feathered), feet (webbed). Features of a concept included by only a single control subject were dropped from the set.

The raters considered the features listed for each item to determine whether they were sufficiently visually similar to one another to be coded as the same visual feature (e.g., the neck on a duck and the neck on a camel). For each feature, a list of modifiers was then formulated that described small differences between otherwise similar features. For example, possible modifiers for neck were curved, thin, long, or short. For some features, several modifiers may be appropriate (e.g., curved and thin), however, in other cases, the modifiers were mutually exclusive (e.g., short and long). Thus, the modifiers long, thin, and curved applied to the neck on the swan as typically drawn; whereas the modifiers long and curved (but not thin) applied to the neck of the camel. Moreover, any object that had a visually similar property (such as the spout on a watering can) would also be scored as having a "neck," with the appropriate modifiers. This scheme allowed us to capture both the broad visual similarities and subtle differences among items with somewhat similar but not identical visual characteristics. Individual features and their modifiers were treated identically in the analyses that follow; we differentiate them here simply to describe how the scoring scheme was employed in the attempt to capture different aspects of visual similarity.

Scoring. Score sheets were constructed by concatenating the full list of features and modifiers. The control drawings were subsequently rescored, with the new score sheets, by identifying each visual feature that was present in the drawing (regardless of whether the visual feature had been listed for the particular object in the first place). For each picture, features were then divided into two types: those that all controls included in their depiction, and features that were inconsistent across controls. Only features produced by all the control subjects were used to evaluate the patients' drawings. Data from the control drawings were also used to classify individual features according to their distinctiveness, as described below.

Scoring the patient data. The features present or absent in the patient drawings were classified in the following way: as correct if it was an appropriate target feature that the patient successfully included in the picture (e.g., correctly putting two legs on a 
duck); as an omission if it was a target property that all control subjects produce but that the patient failed to include (e.g., failing to put a beak on a duck); or as an intrusion if it was a property that the patient incorrectly included in the picture (e.g., putting four legs on a duck).

The category of fruit was excluded from any of the further analyses because one of the patients (IF) failed to produce drawings for all eight exemplars.

Delayed copying of geometric shapes. In order to determine whether the errors observed in delayed drawing reflected impaired semantic memory rather than defective episodic memory, we also asked the subjects to produce delayed copies of geometric shapes. These ranged in complexity from very simple box-like shapes with only two or three features to fairly complex arrangements with many different features (see Appendix A for examples of the geometric shapes). As these stimuli have almost no semantic content, a conceptual impairment should have little effect on copying performance, even with a delay. An episodic deficit, on the other hand, should influence delayed copying of both pictures and geometric figures. The geometric shapes were administered in the same way as the delayed copy- ing of the 64 items: inspection of the target figure, followed by a $10 \mathrm{~s}$ filled delay, followed by an attempt to reproduce the just-seen figure.

A scoring scheme was constructed where 1 point was given for each feature in a design, ranging from 3 to 8 . The designs were then grouped, for the analysis, into designs classified as easy, with 3-4 features $(n=3)$, moderate, with 5-6 features $(n=3)$, and difficult, with $7-8$ features $(n=4)$.

\section{Results}

\section{General neuropsychology}

The three patients covered a range of impairment as indicated by their performance on the MiniMental State Examination (see Table 5). They all showed reasonably intact working memory as measured by forward and backward digit span. All patients exhibited some impairment on the letter fluency test, although this result probably reflects the patients' level of anomia rather than deficits in executive functioning. There was preservation of nonverbal problem-solving skills as measured by Raven's matrices, and of visuospatial skills as assessed by copy of the Rey Figure and various subtests of the Visual Object and Space Perception

Table 5. General neuropsychology

\begin{tabular}{lcccc}
\hline Test (maximum score) & $D S$ & $D C$ & $I F$ & Control mean (SD) \\
\hline MMSE (30) & 23 & 18 & 11 & $28.8(0.5)$ \\
Digit span & & & & \\
Forward & 6 & 7 & 5 & $6.8(0.9)$ \\
Backward & 4 & 2 & 5 & $4.7(1.2)$ \\
Letter fluency (total: FAS) & 7 & 16 & 16 & $44.2(11.2)$ \\
Raven's coloured matrices (percentiles) & 95 & $>95$ & 90 & \\
Rey Figure & & & & \\
Copy (36) & 35 & 32 & 26 & $34(2.9)$ \\
Immediate recall (36) & 17.5 & 12 & $\mathrm{NT}$ & $18.3(5.2)$ \\
VOSP & & & & \\
Incomplete letters (20) & 20 & 20 & $\mathrm{NT}$ & $19.2(0.8)$ \\
Dot counting (10) & 10 & 10 & 10 & $9.9(0.3)$ \\
Object decision (20) & 18 & 17 & 14 & $16.5(3.5)$ \\
Position discrimination (20) & 20 & 17 & 20 & $19.8(0.6)$ \\
Cube analysis (10) & 10 & 10 & 6 & $9.7(2.5)$ \\
Number location (10) & 9 & 10 & $\mathrm{NT}$ & $8.9(2.8)$ \\
\hline
\end{tabular}

The patients are ordered according to their performance on the naming and word-to-picture matching tests (see Table 6). VOSP: Visual Object and Space Perception battery; NT: not tested.

\section{COGNITIVE NEUROPSYCHOLOGY, 2003, 20 (1)}


battery. Notably, performance on the Object Decision subtest of the VOSP was good in all cases. Recall of the Rey Figure in the two cases tested suggests reasonably good nonverbal episodic memory, though DC (the more severe patient) was near the bottom of the control range. Given the complex nature of this geometric figure and the long delay under which its recall is tested, it is not surprising that DC had a mild impairment on this task.

Semantic tests. As shown in Table 6, all three patients showed severely reduced category fluency and a profound degree of anomia on the naming test, but a range of impaired comprehension as measured by the word-to-picture matching task (NB, in Tables 5 and 6 the patients are ordered to reflect decreasing naming and comprehension scores). None of the patients scored at better than chance level on the Concrete and Abstract Word Synonym test, and all three (especially DC and IF) were also impaired on both conditions of the Pyramid and Palm Trees test.

\section{Analysis of drawing data}

Qualitative observations. As in Study 1, the majority of frank misdrawing errors produced by the patients in drawing to name were semantically related to the target concept (e.g., "tiger" $\rightarrow$ lion; "key" $\rightarrow$ door). Cross-domain errors were rare and only observed in the most impaired patient, IF. These errors were scored in the same way as the other items. There were some instances, however, where the patients refused to draw an item because they had no idea what the name meant.

Effect of task condition and disease severity. Figure 2 shows some examples of the drawings produced by the most mildly affected patient, DS. Of primary interest was how performance varied as a consequence of the task condition (immediate copy, delayed copy, or drawing to name), the severity of the semantic deficit (taking DS, DC, and IF as representative of mild, moderate, and severe stages, respectively), and the semantic domain to which each item belongs (animal or artefact). Accordingly, the data were coded with a separate record for each individual drawing; factors that varied between individual drawings were coded as fixed, between-case factors in the analyses of variance described hereafter. There were three factors of interest that varied between individual drawings: the patient who produced the drawing (DS, DC, or IF in order of increasing severity), the task condition in which the drawing was produced (immediate copy, delayed copy, drawing to name), and the semantic domain to which the stimulus item belonged (animal or artefact). In subsequent analyses, we will consider factors that varied within individual drawings, and will treat these as withincase factors.

Table 6. Assessment of semantic memory

\begin{tabular}{lrrrc}
\hline Test (maximum score) & $D S$ & $D C$ & $I F$ & Control mean (SD) \\
\hline Category fluency & 3 & 6 & 2 & $60.3(12.6)$ \\
Living & 10 & 4 & 5 & $54.8(10.3)$ \\
Man-made & 17 & 11 & 1 & $62.3(1.6)$ \\
Naming (64) & 58 & 36 & 18 & $63.7(0.5)$ \\
Word-picture matching (64) & & & & \\
Word synonyms & $12^{\mathrm{a}}$ & $14^{\mathrm{a}}$ & $13^{\mathrm{a}}$ & $23.7(1.3)$ \\
Concrete (25) & $14^{\mathrm{a}}$ & $13^{\mathrm{a}}$ & $13^{\mathrm{a}}$ & $23.0(2.1)$ \\
Abstract (25) & & & & \\
Pyramids and Palm Trees & 46 & $25^{\mathrm{a}}$ & $28^{\mathrm{a}}$ & $51.1(1.1)$ \\
Words (52) & 46 & 36 & $22^{\mathrm{a}}$ & $51.2(1.4)$ \\
Pictures (52) & & & & \\
\hline
\end{tabular}

The patients are ordered according to their performance on the naming and word-topicture matching tests.

${ }^{\mathrm{a}}$ Score not significantly better than expected by chance. 


\begin{tabular}{|l|l|l|}
\hline Immediate copying, & Delayed copying & From name \\
\hline Rhinoceros & &
\end{tabular}

Figure 2. A duck and a rbino drawn by DS in the three conditions.

First, we compared the patients' performance on the three different drawing conditions. We expected performance to reflect the relative reliance of performance in each condition upon conceptual knowledge: best performance (because least such reliance) for immediate copying, intermediate performance for delayed copying, and poorest performance (because greatest reliance on semantic memory) for drawing from name. We also expected to replicate the result of Study 1, that drawing performance (here on delayed copying as well as drawing from name) should reflect the severity of the patient's semantic impairment.

An analysis of variance on the mean number of errors (omissions and intrusions combined) across the three patients showed significant main effects of task, $F(2,425)=96.5, p<.001$, and patient, $F(2$, $425)=49.5, p<.001$, and an interaction between task and patient, $F(4,425)=8.55, p<.001$, see Figure 3 . Pairwise comparisons using a Bonferroni correction confirmed that performance on both delayed copying and drawing from name were worse than immediate copying $(p<.01)$ and performance on drawing from name was worse than delayed copying $(p<.01)$. With regard to the individual patients, DC made more errors than DS, and IF made more errors than $\mathrm{DC}($ all $p<.001)$ confirming that performance worsened with degree of semantic deterioration.
Proportion offeatures omitted. One of the goals of the study was to assess how the number of target features omitted by the patients was affected by the task condition and/or the domain to which the item belonged (see Figure 4). The number of target features varied greatly across the 64 items, leading to different opportunities for omissions in each item. The total number of features omitted was, therefore, expressed as a proportion of the number of possible omissions for each item (equal to the number of target features).

Analysis of variance revealed significant main effects of patient, $F(2,433)=62.71, p<.001$, and task, $F(2,433)=129.41, p<.001$, but not domain, $F(1,433)=1.63$, n.s. Post hoc comparisons between the different tasks indicated that there was a significantly greater proportion of omitted features in drawing from name than in delayed copying $(p<.001)$ and a greater proportion of omissions in both of these conditions compared to immediate copying (both $p<.001$ ). With regard to the individual patients, IF produced fewer target features than $\mathrm{DC}(p<.001)$, who in turn produced fewer than DS $(p<.001)$. There was also a significant interaction between task condition and patient, $F(4,433)=$ $12.07, p<.001$, reflecting the fact that the differences in performance across task increased with semantic impairment. 


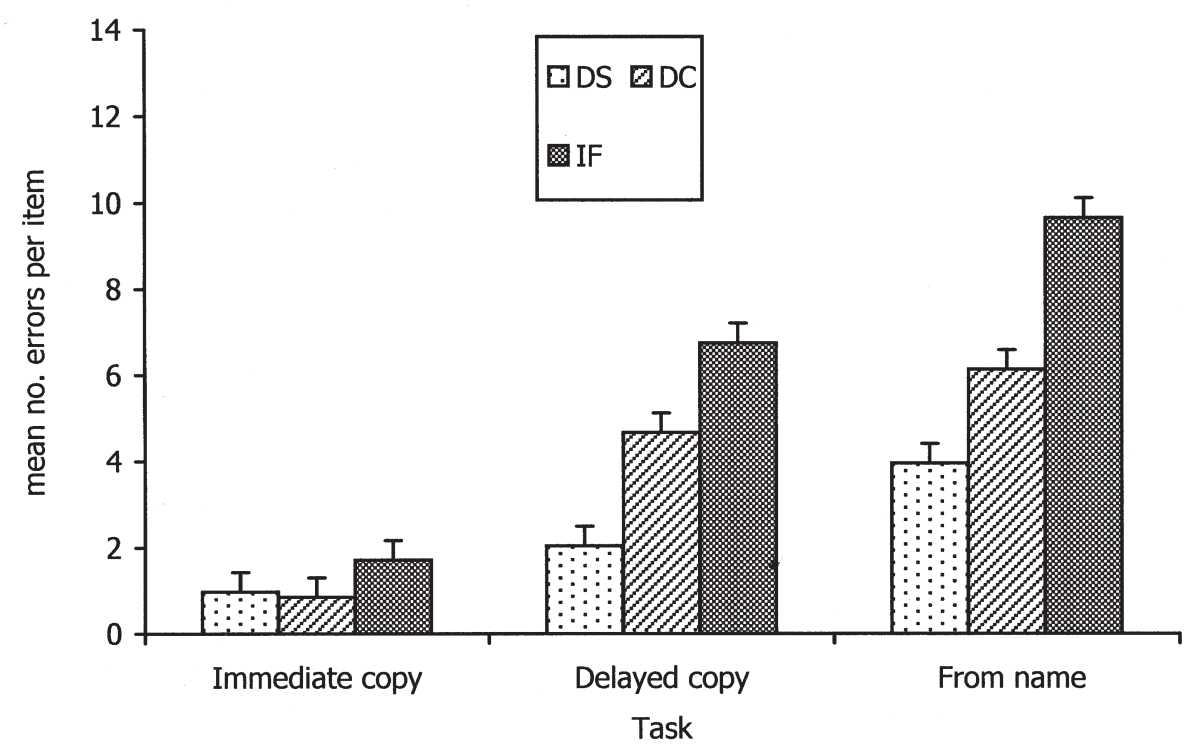

Figure 3. Performance of the three patients on the three task conditions.

Proportion offeatures intruded. Another main goal of Study 2 was to quantify and classify incorrect features that were drawn by the patients (see Figure 5). The number of these intruding errors was calculated as a proportion of the total number of possible features that could be included on each item (as defined by a list of all features included in the control subjects' depictions, collapsed across the two domains). These proportions are fairly small because there are obviously a large number of features that could be included as intrusion errors.

Analysis of variance revealed significant main effects of patient, $F(2,433)=30.64, p<.001$, task, $F(2,433)=54.72, p<.001$, and domain, $F(2,433)$ $=55.65, p<.001$. Post hoc comparisons confirmed that the drawings constructed by IF contained more intrusions than those of DC $(p<.01)$, who in turn produced more of these intruding errors than DS $(p<.01)$. With regard to the different tasks, there were significantly more intrusions in drawing from name and delayed copying than in immediate copying (both $p<.001$ ), but no significant difference between drawing from name and delayed copying $(p>.1)$. Overall, patients' depictions of animals contained more intrusions than the artefacts, $F(1,433)=55.6, p<.001$. There was a significant interaction between domain and task, $F(2,433)=14.8, p<.001$, which is due to the very small proportion of intrusions for concepts from either domain in immediate copying but a much larger and domain-sensitive rate of these errors in the other two conditions. There was also an interaction between patient and task, $F(4,433)=6.52$, $p<.001$, indicating that the difference in intrusions across the tasks increased with level of semantic deficit.

Effects of feature distinctiveness. As discussed in the Introduction, patients with progressive semantic impairments appear to have more robust preservation of knowledge about the general or shared properties of objects, relative to knowledge about more distinctive and idiosyncratic properties (McRae \& Cree, 2002; Rogers et al., 2002a). We predicted that a similar pattern should be observed in the patients' drawing and delayed copying performance. Specifically, a feature that is present in many exemplars of a category should be more likely to be correctly retained than a feature that characterises just one or a few items.

In order to determine the extent to which each property in a given drawing was shared by other 
(a) Immediate copy

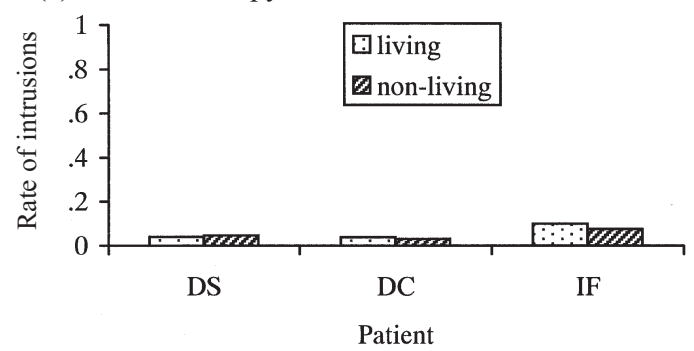

(b) Delayed copy

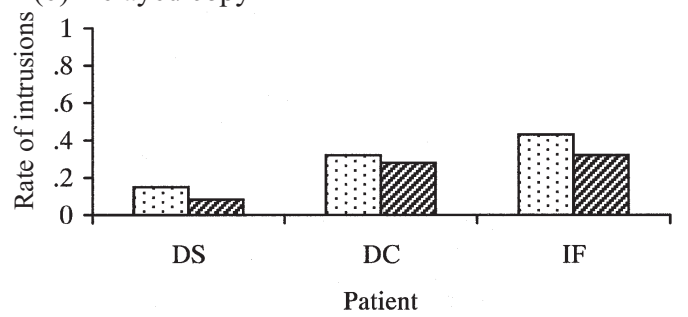

(c) Drawing from name

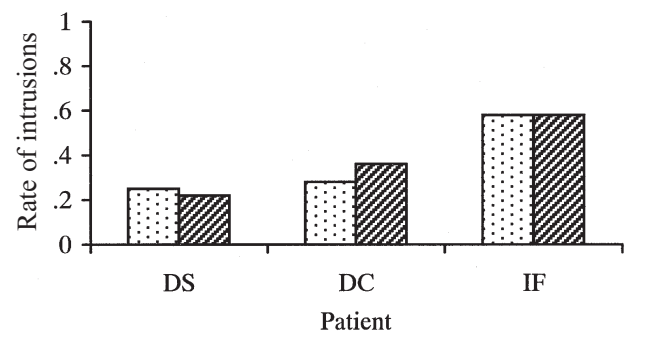

Figure 4. Proportion of features missed in (a) immediate copying; (b) delayed copying; (c) drawing from name. Rate of misses = number of misses/total number of possible missed (the number of target features) per item.

items in the same semantic domain or category, we turned to the drawings produced by our normal control group. We classified each feature in every drawing as either "shared-across-domain," "shared-across-category," or "distinctive," according to a technique similar to that devised by Garrard et al. (2001). For every feature in a given drawing, we calculated a "distinctiveness" score, which described the proportion of items in the same semantic category that also include the feature. For example, if a control subject drew wings on six of the eight birds, the feature "wings" would have a value on the distinctive $\rightarrow$ shared dimension of $6 / 8$,
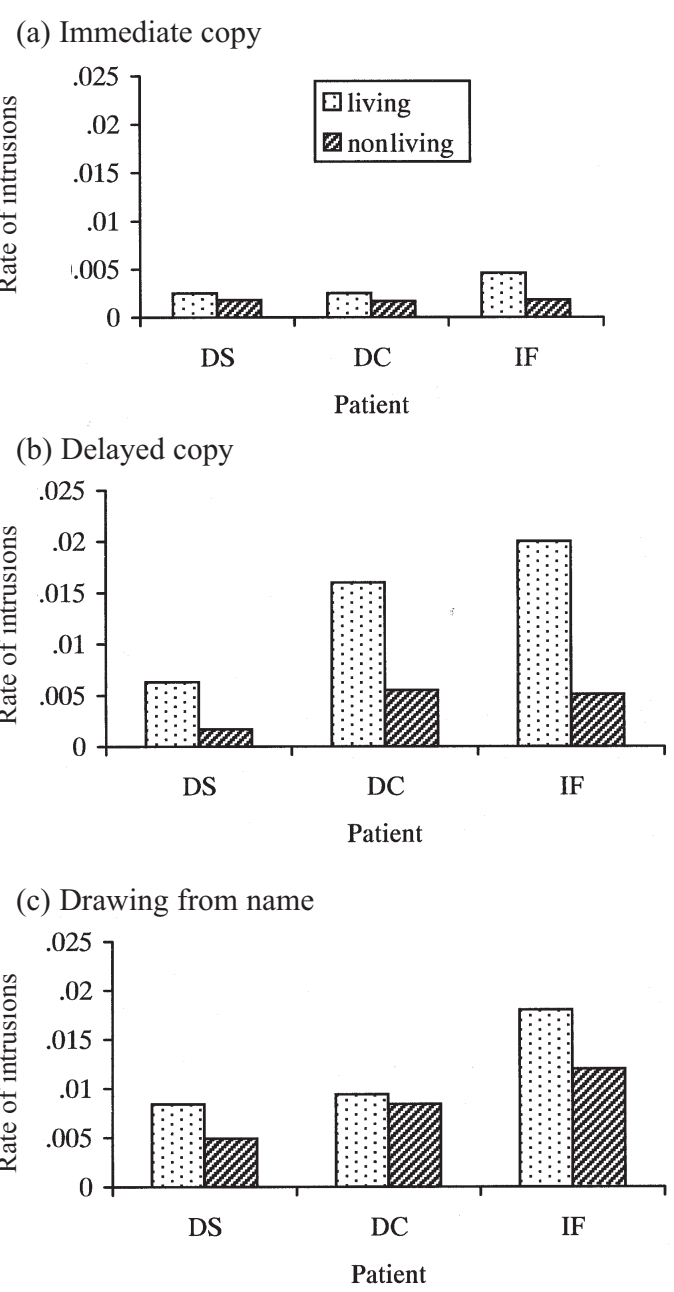

Figure 5. Proportion of false positives in (a) immediate copying; (b) delayed copying; (c) drawing from name. Rate of false positive errors $=$ number of false positive errors/number of possible distractors (see text).

or 0.75 , for that subject and category. Note that distinctiveness for a given property can vary depending upon the category in question; for example "neck" is shared by most of the bird and land animal items, but is quite distinctive among the various artefact categories items, where it is present in just the guitar and watering can.

Distinctiveness was calculated for every feature in all items, for each subject, and across each category. We then averaged across the control subjects to obtain a distinctiveness score for each feature,

\section{COGNITIVE NEUROPSYCHOLOGY, 2003, 20 (1)}


relative to every category. Although this distinctive $\rightarrow$ shared dimension is clearly a continuum, previous studies involving feature databases have found that the distribution of shared and distinctive features across domains is bimodal (Garrard et al., 2001; Rogers et al., 2002a), with modes falling toward the high and low ends of the distribution (i.e., many features shared by most members of a category, and many distinctive features, but few features shared by only half of the items in the category). A useful way of collapsing the data, therefore, is to classify each property as either "distinctive" (with respect to a particular category), if its score falls below 0.5 , and as "shared" otherwise. Under such a classification scheme, drawings of animals and birds were found to have a higher proportion of shared features (mean proportion/item = 0.59), whilst artefacts had more distinctive features (mean proportion/item $=0.29$; see Rogers et al., 2002a).

It should be noted that some properties might be shared across all items in a given semantic domain (e.g., eyes are shared by most animals), whereas others are shared by intermediate categories, but not by the entire domain (e.g., wings are shared by most birds, but not by most animals). Accordingly, the following classification scheme was adopted: Properties that were shared by more than half of the items in all categories of a given domain (e.g., shared by more than half of the birds and more than half of the land animals) were designated "sharedby-domain." Properties that were shared by more than half of the items in an intermediate category, but were not shared-by-domain, were designated shared-by-category. Remaining properties were designated distinctive.

For each drawing, we then calculated the proportion of omissions and intrusions separately for shared-by-domain, shared-by-category, and distinctive features. Each individual picture thus had three "omission" and three "intrusion" scores associated with it. Because each drawing was considered as an individual case in our statistical analyses, feature distinctiveness (shared-by-domain, sharedby-category, or distinctive) was coded as a threelevel within-case factor. This treatment allowed us to use a within-case ANOVA design to determine whether rates of omissions and intrusions differed reliably for distinctive and shared features.

Effect of distinctiveness on omissions. Analysis of variance predicting the omission rate with patient, task, and domain as fixed, between-case factors, and distinctiveness as a within-case factor, showed significant main effects of distinctiveness, $F(2,222)=$ 186.23, $p<.001$, patient, and task (as per earlier analyses accompanying Figure 4 ). The rate of omissions for each patient split by each level of distinctiveness is shown in Figure 6. Post hoc analyses confirmed that distinctive features were omitted more often than features that were shared on any level, $F(1,222)=311.5, p<.001$, and that features that were shared across items in a category were omitted more frequently than features that were shared across the whole domain, $F(1,222)=53.4$, $p<.001$. There was no significant main effect of domain.

These analyses also revealed three interactions: first, between feature distinctiveness and patient, $F(4,442)=3.41, p<.01$, because the most severely impaired patient (IF) displayed greater discrepancies in performance on the different levels of distinctiveness than did the other patients; second, between feature-distinctiveness and task, $F(4,442)$ $=14.52, p<.001$, because the effect of distinctiveness was fairly small in the immediate copy condition and much larger in the other two conditions; and finally between feature-distinctiveness and domain, $F(2,221)=3.88, p<.05$, because few properties were omitted among features that are shared across domain for both animals and artefacts, whereas among features that are shared only within category, more features were omitted for animals than artefacts.

Effect of feature distinctiveness on the rate of intrusion errors. We also predicted that features that are shared across many items should be more likely to intrude incorrectly into drawings of other members of that category or domain. Analysis of variance predicting rate of intrusions from the same between- and within-case factors confirmed a significant main effect of feature-distinctiveness, $F(2,32)=19.12, p<.001$, with contrasts showing 
(a) Immediate copy

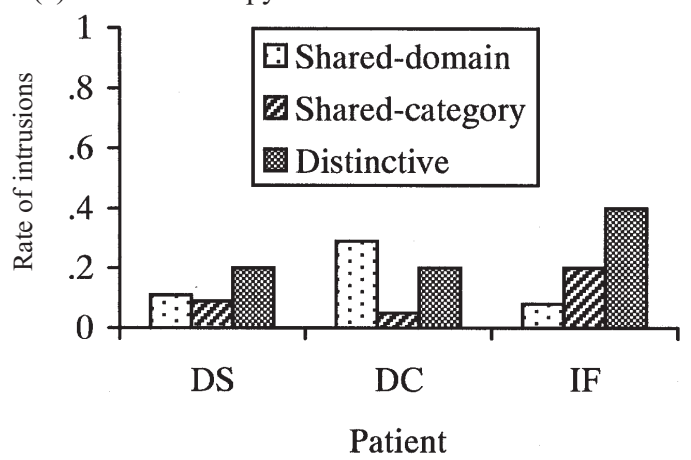

(b) Delayed copy

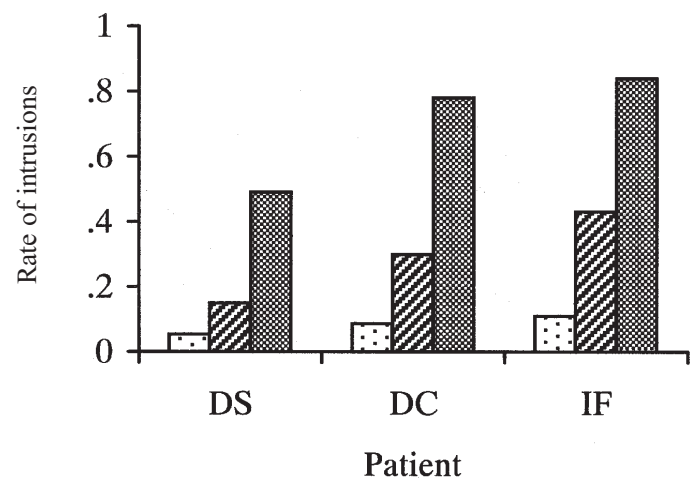

(c) Drawing from name

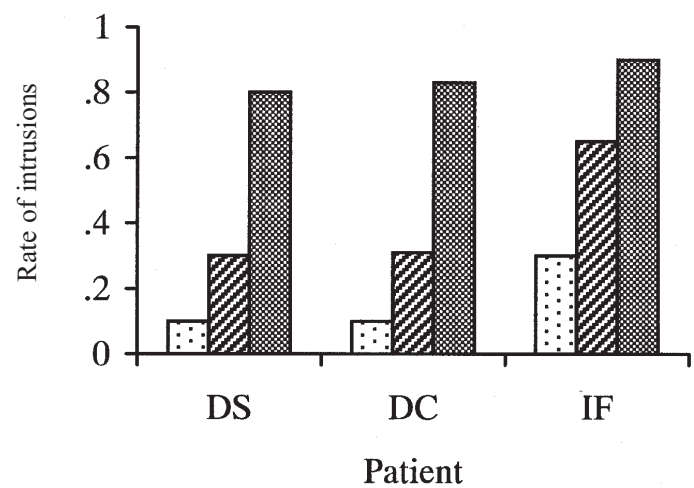

Figure 6. Effect of feature-distinctiveness on (a) the proportion of misses in immediate copying; (b) the proportion of misses in delayed copying; (c) the proportion of misses in drawing from name. Rate of misses $=$ number of misses/total number of possible missed (the number of target features) per item. reliably more intrusions of features shared across domain than features shared across categories $(p<$ .001); and reliably more intrusions for features shared across category than for distinctive properties $(p<.05)$ (see Figure 7). Feature-distinctiveness was found to interact with task, $F(2,33)=4.90, p<$ .05: The effect of distinctiveness was attenuated in the immediate copying task. There were no other significant interactions. Among the between-subjects factors, the only reliable effect was that of task, $F(2,33)=4.95, p<.05$, with contrasts revealing that the delayed copying task and drawing from name elicited more false intrusions than immediate copying (both $p=.058$ ). In contrast to our previous analysis of intrusion errors, domain was not a significant predictor of false positives in this analysis, $F(1,33)$ $=2.94, p>0.1$. That is, the significant difference between animal and artefact items in the rate of intrusion errors reported in the last section disappears when feature distinctiveness is included in the ANOVA.

Delayed copying of geometric shapes. All patients performed within the range of the control subjects on delayed copying of the easy, moderate, and difficult geometric shapes (see Table 7). For simple and moderate shapes, control and patient performance were comparable; for the most complex shapes, patients were at the low end of the control range, indicating that they remembered fewer features than the control subjects.

\begin{tabular}{lcccc} 
Table 7. Performance on delayed copying of the geometric shapes \\
\hline $\begin{array}{c}\text { Easy } \\
\text { shapes } \\
\text { range } \\
\text { (mean) }\end{array}$ & $\begin{array}{c}\text { Moderate } \\
\text { shapes } \\
\text { range } \\
\text { (mean) }\end{array}$ & $\begin{array}{c}\text { Difficult } \\
\text { shapes } \\
\text { range } \\
\text { (mean) }\end{array}$ \\
Group & $N$ & $7-9(8)$ & $9-11(9.7)$ & $16-17(16.7)$ \\
Patients & 3 & $7-9(8.5)$ & $9-13(11.2)$ & $16-27(22.0)$ \\
Controls & 4 & 76
\end{tabular}


(a) Immediate copy

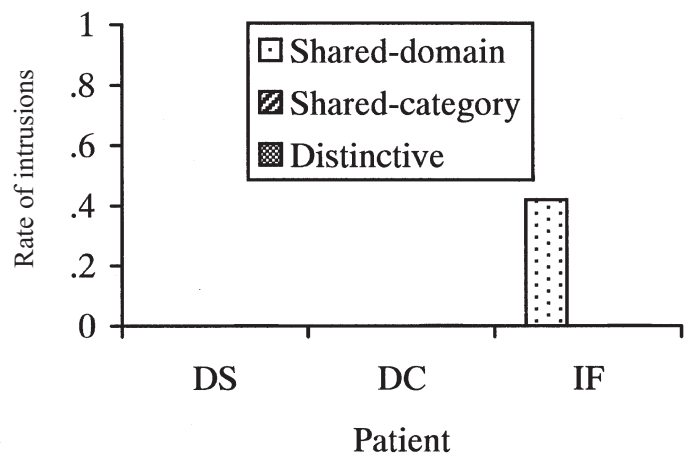

(b) Delayed copy

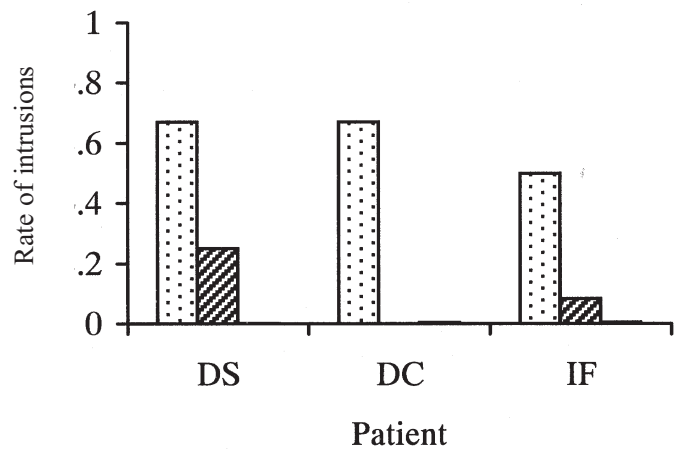

(c) Drawing from name

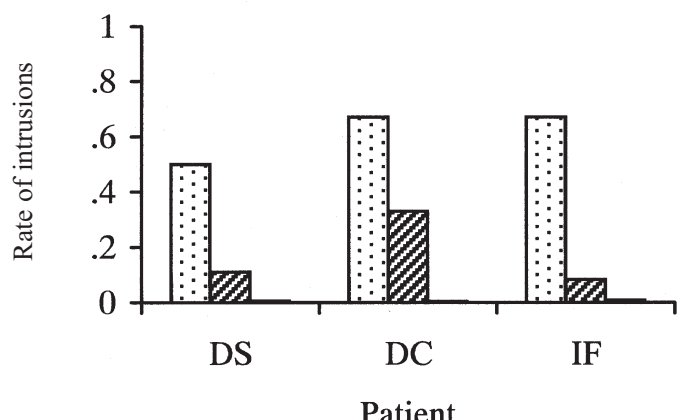

Figure 7. Effect of feature-distinctiveness on the proportion of false positives in (a) immediate copying; (b) delayed copying; (c) drawing from name. Rate of false positive errors $=$ number of false positive errors/number of possible distractors (see text).

\section{DISCUSSION}

The aim of these studies was to investigate the structure of visual conceptual knowledge through featurebased analyses of drawings produced by patients with semantic dementia. In Study 1, the patients' impoverished drawings in response to concept names were characterised by a reduced number of distinctive features for concepts in both living and nonliving domains. We also observed a tendency by the patients to include incorrect features on items from the living domain, although the scoring scheme used in this first experiment did not allow the quantification of these intrusion errors. Analysis revealed significant correlations between performance on drawing, naming, and word-to-picture matching. There was a trend towards better performance on the more familiar items. In Study 2, accuracy of the patients' drawings, whether measured by the total number of errors, the number of omitted features, or the number of intruding features, was significantly influenced by the task condition and the degree of semantic deficit. Performance was always better on immediate copying than delayed copying and, in turn, better on delayed copying, than drawing from name. There was also an interaction between task condition and patient, arising from a decline in performance on delayed copying and drawing from name, but not on immediate copying, as a function of degree of semantic degradation. The rate of intruded features but not of omitted features was influenced by the domain of the item, with a significantly greater proportion of intrusions in the living than the nonliving domain. There was a significant effect of feature-distinctiveness on the proportions of omissions and intrusions. The rate of omitted features was greatest for properties specific to one or very few concepts, and the smallest for features that characterise a large number of concepts within the domain. On the other side of the coin, intruded features were most likely to come from the pool of properties that are shared across domain. 
With respect to differences in performance for animal and artefact items, there are two points of interest to note. First, considering the mean proportion of omissions and intrusions across all visual properties (without regard to property type), omissions were equally likely in both domains, but intrusions occurred more frequently for animals than for artefacts. On the face of it, this finding is consistent with the common hypothesis that semantic knowledge about animals depends to a greater extent upon knowledge of their visual properties: When semantic knowledge is compromised, patients in some sense perform more poorly on the drawing task for animals than for artefacts. Further analyses demonstrated, however, that this apparent difference between domains was eliminated when feature distinctiveness was included as a predictor of intrusion-rate. This suggests that for both domains of items, intrusions in drawing arise from a common cause: Properties that are shared by many semantically related items are more likely to intrude in drawings of objects that do not happen to have them. There are more shared visual properties for animals than for artefacts (McRae \& Cree, 2002; Rogers et al., 2002a), therefore, patients make more intrusions for items in this domain. The results are therefore consistent with theories of semantics within which apparent domain differences can arise from the way that properties are distributed across items in animal and artefact domains (e.g., Devlin et al., 1998; Moss, Tyler, Durrant-Peatfield, \& Bunn, 1998).

In our previous studies, we have demonstrated that the semantic degradation in these patients can be observed in a wide variety of verbal and nonverbal assessments including word-picture matching, picture naming, sound recognition, object use definitions to words and pictures, word and picture sorting, and association tasks (Bozeat et al., 2000; Bozeat, Lambon Ralph, Patterson, \& Hodges, 2002; Hodges, Bozeat, Lambon Ralph, Patterson, \& Spatt, 2000; Hodges et al., 1995; Lambon Ralph, Graham, Patterson, \& Hodges, 1999). The results of this current study indicate that knowledge about the visual properties of concrete objects is also compromised in semantic dementia. Despite good visual perceptual skills and relatively spared episodic and executive functioning, the patients described here were profoundly impaired not only at drawing objects from name but also at copying pictures of objects after a short delay. Taken together with results of the wider body of research cited above, we believe that these data are consistent with the hypothesis that semantic dementia arises from degradation of a unitary amodal semantic system. In the following sections we highlight two aspects of these data that are compatible with this hypothesis.

\section{Consistency of performance across tasks}

Study 1 revealed significant and indeed substantial correlations between the patients' performance on the drawing assessment, object naming, and wordto-picture matching. In all cases, the ability to respond correctly depends on the integrity of conceptual knowledge remaining for the item. The drawing assessments in Study 2 highlighted not only the degree and nature of the conceptual impairment in semantic dementia, but also the selective nature of the deficit in this disorder (Hodges et al., 1992; Warrington, 1975). The patients performed well on immediate copying of the real items and novel geometric figures (including the complex Rey figure), reflecting their preserved visuospatial skills and good praxis. Delayed copying for pictures of real items recruits two additional cognitive systems: episodic and semantic memory. The semantic element in this task can, at least to some extent, be isolated by a comparison with delayed copying of abstract geometric figures: As novel geometric figures have little semantic content, it seems likely that delayed copying accuracy relies almost completely on a visual episodic memory of the displayed pictures. So long as the task requirements were limited to episodic memory, visuospatial skills, and praxis, the patients with semantic dementia were largely within the range of the control subjects. When the task recruited semantic knowledge, however, the selective conceptual impairment in these cases became apparent. In general, the good degree of correspondence in the degree of impairment witnessed in very different kinds of semantic tasks as well as the remarkable 
sparing of performance in nonsemantic tasks, suggest a common underlying cause to the pattern of deficits observed in the disorder.

Consistency of errors across tasks. Second, the pattern of deficits observed in the drawing tasks was similar to that seen in other semantic tasks both visual and verbal. Patient drawing errors were not random, but were structured in ways that relate to the organisation of conceptual knowledge as assessed by other methods. For example, patients were most likely to omit specific and idiosyncratic properties from their drawings, and were least likely to omit properties that are shared across items in the same semantic domain. A similar pattern is observed in picturenaming, definition, and sorting tasks (Hodges et al., 1994, 1995; Lambon Ralph et al., 1999): In all three cases, patients are frequently unable to produce or access the specific details that differentiate objects from their semantic neighbours, but show somewhat better knowledge for the generalities that characterise broad semantic domains. The intrusions in drawing reported here also find a parallel in studies of picture naming: Just as the patients "over-extend" characteristic visual properties of objects in a given category to items that do not happen to share them (e.g., adding four legs to a duck), so too do they over-extend the names of highly familiar or typical items to less familiar items from the same semantic category (Hodges et al., 1995). In short, the pattern of deficits observed in drawing is just another example of general trends apparent across a variety of semantic tasks.

\section{Effects of semantic impairment on structural knowledge}

It has been proposed that information about the visual or structural properties of objects are stored in dedicated representations that are separate from, and function independently of, semantics. This view is supported by studies of patients who, despite impaired access to semantics from vision, nevertheless perform well on tests of visual object recognition such as the object-decision task (Riddoch, Humphreys, Coltheart, \& Funnell, 1988) and hence are thought to have an intact structural description system. If this system is invoked to explain productive as well as receptive aspects of visual object processing, then the current data might be explained by proposing that the patients' poor drawing performance reflects additional impairment to the independent, nonsemantic structural description system.

Nothing in the current data can refute this interpretation. As noted above, however, the pattern of errors observed in drawing, together with good correlations between drawing and other semantics tasks suggest that all deficits result from a single underlying impairment. If the poor performance in drawing results from the impairment of a separate, independent system of visual structural knowledge, it is not clear to us why performance would correlate so well with other verbal tasks or indeed why knowledge of distinctive visual properties should be more vulnerable to impairment than knowledge of spared properties. Our preferred interpretation is that visual-structural representations interact with, and to some extent depend upon, semantic representations, so that the ability to activate or construct a visual representation of an object suffers from reduction of the normal feedback from conceptual knowledge - a hypothesis that is consistent with recent studies of object-decision in semantic dementia (Rogers et al., 2002a; Rogers, Lambon Ralph, Patterson, \& Hodges, 2002b). This interpretation is similar in many respects to the recent theory of object recognition put forward by Humphreys and Forde (2001).

\section{Using drawing as an assessment of semantic memory}

In concluding that the data presented here are compatible with the hypothesis of a unitary (but very distributed) conceptual network, we have tried to emphasise the considerable extent to which results from the drawing task parallel those from other approaches to assessing semantic memory and its disorders. No one of these approaches is superior to another, and each has its disadvantages: For example, drawing provides no perspective on what the patient might know about how an object is used or where it is typically found. On the other hand, 
drawing also has some specific advantages for assessing physical-feature knowledge. In particular, it enables observation of fine gradations of such knowledge: patients may omit or falsely include individual features, thereby indicating a subtle impairment in conceptual knowledge for that particular item. Tests of naming and comprehension require the subject to assign a single label to the concept, thus restricting their responses and forcing them to categorise the item. The delayed copying paradigm provides an assessment of semantic memory, which has no verbal input or output, is relatively quick and easy to administer, requires no complex instructions and provides a very rich data set.

Manuscript received 31 July 2001 Revised manuscript received 9 July 2002 Revised manuscript accepted 28 August 2002

\section{REFERENCES}

Bozeat, S., Lambon Ralph, M. A., Garrard, P., Patterson, K., \& Hodges, J. R. (2000). Non-verbal semantic impairment in semantic dementia. Neuropsychologia, 38, 1207-1215.

Bozeat, S., Lambon Ralph, M. A., Patterson, K., \& Hodges, J. R. (2002). When objects lose their meaning: what happens to their use? Cognitive, Affective, \& Behavioral Neuroscience, 2, 236-251.

Caramazza, A., Hillis, A. E., Rapp, B. C., \& Romani, C. (1990). The multiple semantics hypothesis: Multiple confusions. Cognitive Neuropsychology, 7, 161-189.

Devlin, J. T., Gonnerman, L. M., Andersen, E. S., \& Seidenberg, M. S. (1998). Category specific semantic deficits in focal and widespread brain damage: A computational account. Journal of Cognitive Neuroscience, 10, 77-94.

Folstein, M. F., Folstein, S. E., \& McHugh, P. R. (1975). "Mini-mental state": A practical method for grading the mental state of patients for clinicians. Journal of Psychiatric Research, 12, 189-198.

Franklin, S., Van Sommers, P., \& Howard, D. (1987). Drawing without meaning? Dissociations in the graphic performance of an agnosic artist. In R. Campbell (Ed.), Mental lives: Case studies in cognition. Oxford, UK: Blackwell.

46 COGNITIVE NEUROPSYCHOLOGY, 2003, 20 (1)
Funnell, E. (1995). From objects to properties: Evidence for spreading semantic activation in a case of semantic dementia. Memory, 3, 497-519.

Garrard, P., Lambon Ralph, M. A., Hodges, J. R., \& Patterson, K. (2001). Prototypicality, distinctiveness and intercorrelation: Analyses of the semantic attributes of living and nonliving concepts. Cognitive Neuropsychology, 18, 125-174.

Garrard, P., Patterson, K., Watson, P., \& Hodges, J. R., (1998). Category-specific semantic loss in dementia of Alzheimer's type: Functional-anatomical correlations from cross-sectional analyses. Brain, 121, 633-646.

Graham, K. S., Patterson, K., Pratt, K., \& Hodges, J. R. (1999). Relearning and subsequent forgetting of semantic category exemplars in a case of semantic dementia. Neuropsychology, 13, 359-380.

Hodges, J. R., Bozeat, S., Lambon Ralph, M. A., Patterson, K., \& Spatt, J. (2000). The role of conceptual knowledge in object use: Evidence from semantic dementia. Brain, 123, 1913-1925.

Hodges, J. R., Garrard, P., \& Patterson, K. (1998). Semantic dementia. In A. Kertesz \& D. G. Munoz (Eds.), Pick's disease and Pick complex (pp. 83-104). New York: Wiley-Liss.

Hodges, J. R., Graham, N., \& Patterson, K. (1995). Charting the progression in semantic dementia: Implications for the organisation of semantic memory. Memory, 3, 463-495.

Hodges, J. R., Patterson, K., Oxbury, S., \& Funnell, E. (1992). Semantic dementia: Progressive fluent aphasia with temporal lobe atrophy. Brain, 115, 17831806.

Hodges, J. R., Patterson, K., \& Tyler, L. K. (1994). Loss of semantic memory: Implications for the modularity of mind. Cognitive Neuropsychology, 11, 505-542.

Howard, D., \& Patterson, K. (1992). Pyramids and Palm Trees: A test of semantic access from pictures and words. Bury St. Edmunds, UK: Thames Valley Test Company.

Humphreys, G. W., \& Forde, E. M. E. (2001). Hierarchies, similarity and interactivity in object recognition: "category-specific" neuropsychological deficits. Behavioural and Brain Sciences, 24, 453-509.

Lambon Ralph, M., Graham, K. S., Patterson, K., \& Hodges, J. R. (1999). Is a picture worth a thousand words? evidence from concept definitions by patients with semantic dementia. Brain and Language, 70, 309-335.

Lambon Ralph, M. A., \& Howard, D. (2000). Gogi aphasia or semantic dementia? Simulating and assessing poor verbal comprehension in a case of progressive 
fluent aphasia. Cognitive Neuropsychology, 17, 437465.

Lambon Ralph, M. A., Howard, D., Nightingale, G., \& Ellis, A. W. (1998). Are living and non-living category-specific deficits causally linked to impaired perceptual knowledge? Evidence from a categoryspecific double dissociation. Neurocase, 4, 311-338.

Lambon Ralph, M. A., Patterson, K., \& Hodges, J. R., (1997). The relationship between naming and semantic knowledge for different categories in dementia of Alzheimer's type. Neuropsychologia, 35, 1251-1260.

McRae, K., \& Cree, G. S. (2002). Factors underlying category-specific semantic deficits. In E. M. E. Forde \& G. Humphreys (Eds.), Category specificity in mind and brain. Hove, UK: Psychology Press.

Moss, H. E., Tyler, L. K., Durrant-Peatfield, M., \& Bunn, E. M. (1998). Two eyes of a see-through: Impaired and intact semantic knowledge in a case of selective deficit for living things. Neurocase, 4, 291-310.

Raven, J. C. (1962). Coloured Progressive Matrices Sets A, $A B, B$. London: H. K. Lewis.

Raven, J. C. (1965). Advanced Progressive Matrices Sets I E II. London: H. K. Lewis.

Rey, A. (1941). L'examen psychologique dans les cas d'encephalopathie traumatique. Arch Psychologie, 28, 286-340.

Riddoch, M. J., Humphreys, G. W., Coltheart, M., \& Funnell E. (1998). Semantic systems or system? Neuropsychological evidence re-examined. Cognitive Neuropsychology, 5, 3-25.

Rogers, T. T., Lambon Ralph, M. A., Patterson, K., Bozeat, S., McClelland, J. L., \& Hodges, J. R. (2002a). The structure and deterioration of semantic memory: A neuropsychological and computational investigation. Psychological Review.
Rogers, T. T., Lambon Ralph, M. A., Patterson, K., \& Hodges, J. R. (2002b). Object recognition under semantic impairment: the effects of conceptual regularities on perceptual decisions. Language and Cognitive Processes.

Rosch, E. H. (1973). On the internal structure of perceptual and semantic categories. In T. E. Moore (Ed.), Cognitive development and acquisition of language. New York: Academic Press.

Sartori, G., Job, R., Miozzo, M., Zago, S., \& Marchiori, G. (1993). Category-specific form-knowledge deficit in a patient with Herpes Simples virus encephalitis. Journal of Clinical and Experimental Neuropsychology, 15, 280-299.

Snodgrass, J. G., \& Vanderwart, M. (1980). A standardized set of 260 pictures: Norms for name agreement, image agreement, familiarity, and visual complexity. Journal of Experimental Psychology: Human Learning and Memory, 6, 174-215.

Warrington, E. K. (1975). Selective impairment of semantic memory. Quarterly Journal of Experimental Psychology, 27, 635-657.

Warrington, E. K., \& James, M. (1991). The Visual Object and Space Perception Battery. Bury St. Edmunds, UK: Thames Valley Test Company.

Warrington, E. K., McKenna, P., \& Orpwood, L. (1998). Single word comprehension: A concrete and abstract word synonym test. Neuropsychological Rehabilitation, 8, 143-154.

Warrington, E. K., \& Shallice, T. (1984). Category specific semantic impairments. Brain, 107, 829-854.

Wechsler, D. A. (1987). Wechsler Memory ScaleRevised. San Antonio, TX: Psychological Corporation. 